\title{
Electrochemical Sensor Based on poly(3,4-ethylenedioxy - thiophene) Doped with Transition Metals for Detecting Rutin in Buck Wheat Tea
}

\author{
Limin Lu ${ }^{1,2}$, Liping $\mathrm{Wu}^{1}$, Wenmin Wang ${ }^{1}$, Xiaoyi Long ${ }^{1}$, Jingkun $\mathrm{Xu}{ }^{2, *}$, and Haohua He ${ }^{1, *}$ \\ ${ }^{1}$ Institute of functional materials and agricultural applied chemistry, College of Science, Jiangxi \\ Agricultural University, Nanchang 330045, PR China \\ 2 Jiangxi Key Laboratory of Organic Chemistry, Jiangxi Science and Technology Normal University, \\ Nanchang 330013, PR China \\ *E-mail: xujingkun@tsinghua.org.cn, hhhua64@163.com
}

doi: $10.20964 / 2018.02 .66$

Received: 15 June 2017 / Accepted: 18 August 2017 / Published: 28 December 2017

Herein, poly(3,4-ethylenedioxythiophene) (PEDOT) films doped with transition metals were electrochemically synthesized. Transition metal ions-EDTA (M-EDTA, $\mathrm{M}=\mathrm{Cu}^{2+}, \mathrm{Mn}^{2+}, \mathrm{Fe}^{3+}, \mathrm{Co}^{2+}$, $\mathrm{Ni}^{2+}$ ) complexes served as dopants. Rutin as model molecular was utilized to investigate its electrochemical responses at PEDOT/M-EDTA modified electrodes. It was found the PEDOT/Co ${ }^{2+}$. EDTA exhibited better electrocatalytic activity toward rutin oxidation than other PEDOT/M-EDTA modified electrodes. Thus, PEDOT/Co ${ }^{2+}$-EDTA film-based electrochemical sensor was used to detect rutin. Low detection limit $(1.67 \mathrm{nM})$, good reproducibility and wide linear range $(5 \mathrm{nM}-100 \mu \mathrm{M})$ were obtained. The sensor was employed for the determination of rutin in buckwheat tea. These results suggest that PEDOT/Co ${ }^{2+}$-EDTA could be utilized as a promising sensing platform for rutin detection.

Keywords: PEDOT; Transition metals; Rutin; Electrochemical detection; Sensor

\section{$\underline{\text { FULL TEXT }}$}

(C) 2018 The Authors. Published by ESG (www.electrochemsci.org). This article is an open access article distributed under the terms and conditions of the Creative Commons Attribution license (http://creativecommons.org/licenses/by/4.0/). 